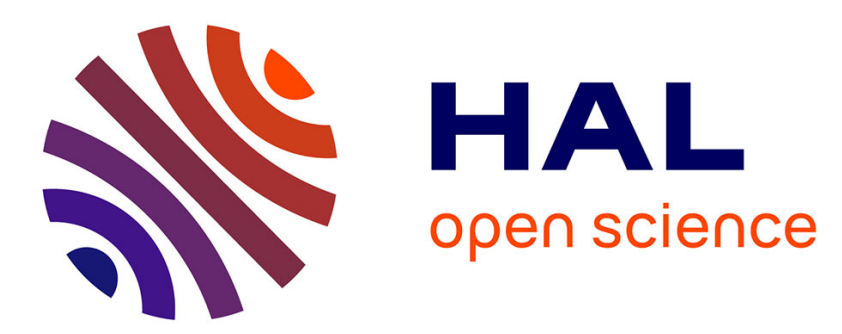

\title{
Créer des espaces pour la création artistique et la médiation culturelle et civique : les maisons des jeunes artistes indépendants bucarestois
}

Romina Surugiu

\section{- To cite this version:}

Romina Surugiu. Créer des espaces pour la création artistique et la médiation culturelle et civique : les maisons des jeunes artistes indépendants bucarestois. Culture et Musées, 2018, 31, pp.91-116. 10.4000/culturemusees.1817 . hal-03261945

\section{HAL Id: hal-03261945 \\ https://hal.science/hal-03261945}

Submitted on 16 Jun 2021

HAL is a multi-disciplinary open access archive for the deposit and dissemination of scientific research documents, whether they are published or not. The documents may come from teaching and research institutions in France or abroad, or from public or private research centers.
L'archive ouverte pluridisciplinaire HAL, est destinée au dépôt et à la diffusion de documents scientifiques de niveau recherche, publiés ou non, émanant des établissements d'enseignement et de recherche français ou étrangers, des laboratoires publics ou privés. 


\section{OpenEdition} Journals

\section{Culture \& Musées}

Muséologie et recherches sur la culture

$31 \mid 2018$

Entre création et médiation : les résidences d'écrivains et d'artistes Dossier

\section{Créer des espaces pour la création artistique et la médiation culturelle et civique : les maisons des jeunes artistes indépendants bucarestois}

Create spaces for artistic creation and cultural and civic mediation : the houses of young independent Bucharest artists

Crear espacios para la creación artística y la mediación cultural y cívica: las casas de jóvenes artistas independientes de Bucarest

Romina Surugiu

p. $91-116$

https://doi.org/10.4000/culturemusees.1817

\section{Résumés}

Français English Español

Le sujet de la recherche est le cas des maisons des jeunes artistes indépendants, créées à Bucarest, capitale de la Roumanie. Il s'agit d'espaces hybrides, alternatifs considérés par leurs résidents artistes plasticiens, architectes, acteurs, activistes civiques, journalistes, photographes etc. - comme " domestiques-créatifs »: destinés au logement, à la production artistique et culturelle et à la médiation culturelle et civique, en même temps. Ces maisons offrent des résidences permanentes ou temporaires pour les jeunes créateurs et sont des espaces pour des expositions, débats, projections de films, spectacles de théâtre et fêtes.

Cette contribution présentera le contexte de l'apparition et du développement des maisons des artistes à Bucarest. Les maisons des artistes seront investiguées comme emplacement, structure d'accueil, type de financement, projet de création et de médiation culturelle. 
This paper's aim is to present a case-study on the houses of young independent artists, established in Bucharest, capital of Romania. These houses are hybrid, alternative spaces. Their residents their residents - visual artists, architects, actors, civic activists, journalists, photographers, etc. - describe them being as « domestic-creative spaces »: with a multiple use : accommodation for artists, spaces for artistic/cultural production and programs of cultural and civic mediation. These houses offer permanent or temporary residence for young artists and they host exhibitions, debates, film screenings, theater performances and festivals.

The present contribution will present the global and national context of the appearance and development of houses of artists in Bucharest. These houses will be investigated as location, organization, funding, artistic aim and cultural mediation.

El tema de investigación es el caso de las casas de jóvenes artistas independientes, creadas en Bucarest, la capital de Rumania. Se trata de espacios híbridos, alternativos, considerados por sus residentes: artistas visuales, arquitectos, actores, activistas cívicos, periodistas, fotógrafos, etc., como espacios "domésticos-creativos": destinados a la vivienda, la producción artística y cultural y la mediación cultural y cívica, al mismo tiempo. Estas casas ofrecen residencias permanentes o temporales para jóvenes creadores y son espacios para exposiciones, debates, proyecciones de películas, espectáculos teatrales y fiestas.

Este artículo presentará el contexto de aparición y de desarrollo de las casas de artistas en Bucarest y serán investigadas como sitio, estructura de acogida, tipo de financiamiento, proyecto de creación y de mediación cultural.

\section{Entrées d'index}

Mots-clés : maisons des artistes, résidence, espace créatif, médiation culturelle, alternatif

Keywords: houses of artists, artistic residence, creative space, cultural mediation, alternative

Palabras clave: casas de artistas, residencia, espacio creativo, mediación cultural, alternativa

\section{Notes de la rédaction}

Manuscrit reçu le 3 septembre 2017

Version révisée reçue le 25 juin 2018

Article accepté pour publication le 7 juillet 2018

\section{Texte intégral}

Andreea Toma est une jeune peintre roumaine diplômée en beaux-arts de l'Université Nationale des Arts de Bucarest. Son travail, qui combine des éléments d'art conceptuel, de pop art et de réalisme, sous le titre media, a été exposé pour la première fois en octobre 2016 à la maison Carol 53. Pour elle, ce fut une occasion inespérée de rencontrer un public attentif au phénomène artistique et de commencer à connaître une célébrité médiatique. Les journalistes de télévision présents lors de l'événement intitulé « Nuit des maisons ", l'ont interviewée. "Je suis sortie de la mer des inconnus, même si c'est à $2 \% \ldots »^{1}$, déclarait-elle à propos de cette expérience.

2 Organisée pour la première fois en septembre 2014, "La Nuit des maisons » a ouvert ses portes au public bucarestois dans un nouveau territoire, peu exploré : un espace hybride, qui cadre difficilement avec le paradigme culturel dominant en Roumanie. Ce lieu alternatif, situé au centre de Bucarest, comprend plus de dix maisons d'artistes organisées sans soutien institutionnel. Elles offrent des résidences permanentes ou temporaires pour les jeunes créateurs et sont, en même temps, des espaces pour des expositions, débats, projections de film, spectacles de théâtre et fêtes.

3 Leurs résidents sont des jeunes créateurs roumains : artistes plasticiens, photographes, architectes, acteurs, journalistes, spécialistes de la communication, etc. L’invitation inaugurale ${ }^{2}$ à l'événement faisait figurer ces demeures sur une carte du ciel, les étoiles étant les maisons, dessinant une sorte de structure symbolique des liens tissés entre elles. Le texte qui accompagnait le dessin était plus qu'une invitation : c'était un document de type manifeste culturel. Il racontait que dans les dernières années, pour surmonter les difficultés causées par " des autorités hostiles » et par " un environnement social apathique » les jeunes artistes de Bucarest se sont organisés dans des communautés de 
création et d'activisme social et civique. Les espaces privilégiés de ces communautés sont les maisons des artistes, définies par leurs habitants comme « espace de vie, de travail artistique, d'exposition et d'événements culturels et civiques»3.

4 Notre recherche se propose d'étudier le cas des maisons des jeunes artistes indépendants de Bucarest comme espaces pour les résidences créatives ainsi que pour la médiation culturelle et civique. Nous présenterons et analyserons le contexte culturel et social de l'apparition et du développement des résidences créatives à Bucarest ; leurs emplacement, structure d'accueil, type de financement, les projets de création, de médiation culturelle et la relation avec un public qui participe aux événements mis en place en coopération par les artistes résidents (d'architectes, de peintres, d'activistes sociaux, journalistes, etc.)

5 Notre hypothèse est que, en concevant ces maisons pour la création artistique, les jeunes artistes indépendants de Bucarest ont eu l'intention d'exprimer une critique artiste et sociale (au sens de Boltanski \& Chiapello, 1999) et de proposer une modalité d'organisation de lieux créatifs, au-delà des institutions formelles roumaines. Dans ce contexte, on considère que la médiation - définie comme lien avec le public - n'est pas seulement un instrument de reconnaissance de la valeur artistique, mais surtout une possibilité d'exercer cette fonction critique et de la rendre plus visible dans la société.

Pour comprendre la réalité des maisons des artistes, complexe et très différente d'autres enjeux artistiques roumains, nous avons mené une enquête de terrain entre septembre 2015 et août 2017. L'enquête a combiné des entretiens semi-directifs avec des artistes résidents et un architecte, des observations non-participantes aux événements organisés par les maisons et une analyse de documents (dépliants, textes de presse ou photos, posts sur réseaux sociaux). Les résultats sont présentés sous la forme d'étude de cas, décrit par la littérature académique comme « une forme de combinaison [des données de recherche] particulièrement fructueuse » (De Sardan, $2014:$ 73). Le produit final de l'étude de cas est une sorte de " portrait culturel » (Creswell, $2013: 96$ ) du groupe de jeunes artistes indépendants roumains censé présenter leurs objectifs et besoins et le changement social qu'ils proposent. Nous avons également intégré l'enjeu de go native autochtones -, (Creswell, 2013 : 96), en investiguant les résidences d'artistes (Villagordo, 2012).

\section{Quelques notes sur le contexte}

Nous intégrons à notre analyse une notion très influente à partir des années 2000, celle de « ville créative », qui s'inscrit dans la logique d'une réorganisation globale du paysage urbain, dans la société postindustrielle. La dissolution des rapports industriels classiques et les changements sociaux apportés par une nouvelle vision du travail ont configuré un " nouvel esprit du capitalisme » (Boltanski \& Chiapello, 1999), c'est-à-dire une construction symbolique soutenue par une forte idéologie de la créativité, de l'authenticité, de la flexibilité qui commençait déjà à être théorisée dans les années 1960. Comme l'expliquent Boltanski et Chiapello (1999 : 244-245), 1968 a permis l'émergence d'une critique sociale qui dénonçait le pouvoir hiérarchisé, le paternalisme, l'autoritarisme, le taylorisme, en promouvant l'émancipation et la liberté d'expression avec des formes empruntées à l'expression artistique. Selon eux, le capitalisme a trouvé des formules pour encapsuler la critique sociale et artistique dans sa structure idéologique. Un outil social très efficace, à l'époque contemporaine, est la créativité, vue comme condition sine qua non du travail innovateur et qualitatif, ainsi que du renouvellement des villes.

8 Dans ce paradigme de la créativité comme impératif contemporain s'inscrit la « ville créative ». Au niveau mondial, la contribution la plus connue, grâce à une très forte campagne de marketing, est celle de Richard Florida, géographe américain, auteur d'ouvrages très médiatisés comme The Rise of the Creative Class (2002), The Flight of Creative Class (2004) ou Who's Your City (2008). Richard Florida met en évidence les 
relations entre la classe créative - l'âme de la ville créative - et le développement économique d'une certaine région urbaine. Le lieu par excellence pour le développement de la classe créative est, selon Florida, la cité, considérée comme un centre de créativité et un incubateur pour l'innovation. (Florida, $2002: 288$ ). Florida note que les communautés doivent s'impliquer dans le développement des industries créatives pour permettre à l'économie locale de se développer (ibid., 2002 : 283). Il est important pour les communautés d'y attirer et de faire s'y établir des artistes et d'autres personnes créatives (Florida, 2008 : 101).

9 La critique faite à Florida, qui semble avoir une portée explicative plus importante que la thèse même de l'auteur, concerne beaucoup d'aspects, notamment l'existence réelle d'une classe créative, le lien entre cette classe créative et le développement des villes réalité ou simple juxtaposition ? -, la conceptualisation floue de la créativité, la relation de cause à effet entre la créativité et la cohésion sociale, l'originalité de la démarche floridienne... (Tremblay \& Tremblay, 2010/2016). Les recherches mentionnées par Darchen et Tremblay (2010/2016), soulignent que la thèse de Florida constitue une " interprétation simplifiée de la croissance économique en milieu urbain » et que les personnes qui font partie de la classe créative ne sont pas nécessairement attirées par les centres urbains. Mais, comme le constatent les auteurs, " cela n'empêche pas la thèse de Florida de servir de référence quant à l'orientation de certaines politiques de développement économique ». En effet, la forme accessible de la thèse floridienne et l'incessant bruit mené autour d'elle ont poussé les municipalités importantes d'Amérique du Nord à adopter cette thèse dans leur plan de développement.

La culture, constate Vivant (2009 : 12), est mentionnée dans les documents de politiques urbaines et devient un véritable « outil de valorisation d'espace ». La valorisation d'espace peut prendre des formes diverses. Vivant (2009:22), par exemple, mentionne les espaces in - formels, institutionnels, reconnus par le monde artistique et par la société - et les lieux artistiques off - sans place bien déterminée sur le marché des biens culturels, sujets à précarité en ce qui concerne les conditions de vie et de travail des artistes, marginaux et alternatifs -. Le public contemporain fréquente et apprécie en même temps les espaces in et les scènes artistiques off, observe Vivant (2009: 24).

11 La médiation culturelle occupe une place très importante dans les activités circonscrites aux espaces artistiques in et off. En analysant des résidences des artistes pour les écrivains, Carole Bisenius-Penin a constaté que cette médiation constitue pour les créateurs " une forme de reconnaissance de leur posture, sorte de consécration de par l'action médiane de la structure culturelle qui effectue une sélection d'auteur accréditant ainsi la valeur littéraire de chaque résident et renforçant au passage sa posture coconstruite, à la fois par la production et par les diverses médiations autour de lui en lien avec les publics » $(2015: 22)$.

12 On note aussi que la figure de l'artiste a connu des mutations importantes à l'époque contemporaine, entre excentricité comme moteur des changements esthétiques, engagement politique et marginalité comme rejet des institutions (Heinich, 2005). Le travail dans les industries créatives est parfois précaire, isolé, difficile à gérer par l’individu et mal rétribué (Hesmondhalgh \& Baker, 2011). La situation devient paradoxale : il est socialement désirable de travailler dans le domaine créatif, mais il est également difficile de s'y affirmer et d'y gagner sa vie. En Roumanie, en particulier, les conditions sociales de production artistique indépendante d'aujourd'hui incluent l'héritage communiste dans l'organisation institutionnelle de la création artistique, c'est-à-dire la conception de l'artiste en tant qu' « artiste d'État » (Cârneci, 2013 ; Macrea-Toma, 2009) et l'apathie sociale qui caractérise les jeunes roumains « les blasés » (Frunzaru \& Ivan, 2011).

\section{Les maisons : création et critique}


Les jeunes créateurs rencontrent des difficultés à chaque pas, partout dans le monde : la difficulté de trouver un atelier, de payer le loyer, de côtoyer ou de faire partie d'une communauté d'artistes vivante, de peindre, de sculpter à la maison ou de pratiquer un instrument chez soi. Les maisons des artistes sont apparues à Bucarest face à la nécessité de leurs résidents de surmonter les difficultés inhérentes à l'entrée dans la carrière artistique et de faire partie d'un « réseau de coopération » ou d'une « action collective » (au sens de Becker, 1999 : 99). Elles sont, comme l'explique Andrei Eugen Lakatos, architecte et professeur d'architecture, des " interventions ad hoc ", des " sites non officiels » des « lieux d'une telle exubérance » 4 qui permettent aux jeunes de créer, sans avoir un appui institutionnel. Comme l'indique le programme-manifeste mentionné plus haut, il leur parait fondamental, pour le bon fonctionnement de ces maisons, de ne pas avoir un appui institutionnel, parce qu' « [i]l est facile d'étouffer une institution mais un réseau informel est très résistant tant que ses communautés sont vivantes 5 ». La communauté des artistes d'une maison réunit des créateurs divers : artistes plasticiens, architectes, photographes, musiciens, acteurs, journalistes, et même inventeurs. Dans cette configuration nous utilisons l'expression " maisons des artistes » dans un sens générique. Leurs résidents et collaborateurs les appellent tout simplement « les maisons ». La consécration de jeunes artistes est favorisée par leur acceptation dans le groupe de créateurs et dans les espaces alternatifs, à la suite d'un examen collégial.

Un des traits définitoires de ces maisons est le caractère hybride. Il s'agit d'une communauté réelle qui coexiste avec une communauté virtuelle - comme dans le cas de Casa jurnalistului - La Maison du journaliste -, d'un espace artistique qui est aussi un espace privé (le cas de Home Mătăsari, de Carol 53 et d'autres résidences) et de l'esprit artistique qui se conjugue avec l'esprit civique (comme dans le cas de Colivia-La Cage). Iulian Văcărean, l'un des fondateurs de Home Mătăsari, explique : « Ce sont des maisons underground, c'est-à-dire [un lieu de] manifestations culturelles autres que celles que le public connait à l'Opéra, à l'Athénée, ou dans les salles d'exposition. Ici se produit un acte artistique qui est très proche du public participant. Elles sont les maisons vivantes de Bucarest, où naît la forme culturelle qui va prendre vie dans cette cité. ${ }^{6}$

Une caractéristique clé de ces maisons est la matérialité de l'espace. Ce n'est pas une communauté virtuelle, construite autour d'une plateforme numérique, mais une communauté « en chair et en os » qui s'est développée autour de ces maisons, habitées par plusieurs artistes indépendants. Nous avons donc relevé l'accent mis sur cette matérialité et sur le caractère privé des bâtiments. Il s'agit de vieilles maisons ou d'appartements construits avant 1945. Colivia-La cage propose, par exemple, « la culture jeune dans une maison de la vieille bourgeoisie ». Les maisons devenues espaces créatifs gardent l'architecture originale et l'aspect général bourgeois. L'intérieur a été restauré, en conservant, autant que possible, des éléments décoratifs tels que poêles en céramique, rampes d'escalier, panneaux ou ornements sur les murs et les plafonds.

Cette préférence pour les demeures bourgeoises, situées dans le centre de la capitale mérite d'être explicitée. Une alternative aurait été les appartements des bâtiments construits après 1945, dans les quartiers dits de la classe ouvrière. Ces bâtiments et appartements que l'on appelle de manière péjorative « communistes » en langage courant, sont explicitement rejetés comme option d'hébergement ou de création pour les artistes : " les loyers y sont très élevés et il n'y a pas suffisamment d'espace », comme l'explique dans un entretien, l'un des résidents de Casa Jurnalistului. Andreea Toma, une jeune peintre, ajoute : « en tant qu'artiste, je suis réticente à quitter le centre-ville car, ici, il y a des galeries d'art, des expositions, des personnes intéressées par cela. On se nourrit de cet environnement, on s'inspire dans la création, parce qu'on n'habite pas dans une maison ordinaire, qui appartient à l'architecture moderne qui est tellement simpliste. La maison a une charge d'énergie que nous, en tant qu'artistes, utilisons dans l'activité créatrice. »

17 Une autre possibilité aurait été l'orientation des artistes vers l'espace ex-industriel, comme c'est le cas des hubs créatifs qui ont un but commercial clairement assumé comme Nod Makerspace à Bucarest ou Fabrica de pensule à Cluj-Napoca -. Les 
recherches menées dans la Roumanie contemporaine ont montré que l'espace exindustriel est abandonné et se dégrade jour après jour. Il existe peu d'opérations de récupération de l'espace ex-industriel (Lakatos, $2017: 138$ ). Comme explique Lakatos, qui a fait des recherches sur le potentiel de ces espaces : « La zone centrale de Bucarest offre quelque chose que les sites ex-industriels - qui ne sont pas démolis - ne peuvent malheureusement pas offrir comme infrastructure, avec tout ce que cela implique : l'accessibilité, les voies de transport, la visibilité, l'interaction. [...] Pour exister, les artistes ont besoin de visibilité et d'un public qui puisse les suivre. Le centre de la ville soutient ces interactions sociales, en étant par définition un espace qui facilite les échanges culturels et, pourquoi pas, le commerce. En ce qui concerne l'option pour des vieilles maisons, je pense que cette tendance vient au moins de deux directions : l'une pragmatique - les vieilles maisons existent déjà, ce sont des espaces déjà construits, et leur aménagement coûte moins que la construction de nouveaux -, et l'autre, d'ordre poétique - les vieilles maisons portent avec elles une histoire (de vie), une certaine émotion, qui peut devenir à un moment donné une partie de la création artistique, ou, tout au moins, un soutien. »7

$\mathrm{Au}$ niveau culturel, nous pouvons également parler d'une sorte d'élimination symbolique de la période communiste de la vie artistique contemporaine - une annihilation par l'absence d'une quelconque mémoire artistique ${ }^{8}$. Les jeunes artistes rejettent les institutions formelles, c'est-à-dire les unions de création qui ont joué un rôle important pour attirer et contrôler les artistes dans l'État communiste (voir Cârneci, 2013 ; Macrea-Toma, 2009).

Le financement provient du crowdfunding, - financement participatif -, des projets artistiques réalisés par les associations à but non lucratif ou de l'argent gagné par les résidents de leur travail artistique ou d'autre type. Il offre un style de vie décrit par un des habitants comme low cost (Surugiu, 2017), mais qui encourage le travail artistique. Dans le cadre de ces maisons, il n'existe pas de programmes locaux ou gouvernementaux de financement pour la création. Cependant, dans d'autres contextes plus officiels, des bourses sont offertes par diverses institutions publiques. Il existe un appui institutionnel pour les créateurs consacrés qui appartiennent aux unions de créations artistiques reconnues - comme l'Union des écrivains, l'Union des architectes et l'Union des artistes plastiques -réminiscences du système communiste. Mais les jeunes artistes ont des difficultés à s’intégrer dans ce système qui est parfois clientéliste ou simplement mal organisé. Un des artistes interviewés pour cette recherche, qui nous a demandé de rester anonyme, nous a donné comme exemple de mauvaise organisation le programme de financement d'ARCUB, le Centre Culturel de Bucarest, qui attribue un budget très réduit pour les jeunes artistes et favorise seulement les grands projets artistiques.

Les maisons sont louées par les artistes dans le respect des conditions légales. Les loyers sont inférieurs à ceux pratiqués dans la zone centrale de Bucarest. Il existe aussi des propriétaires qui acceptent de les céder gratuitement pour un laps de temps limité. Les contextes sont divers, mais dans tous les cas il s'agit d'un problème important : les propriétaires n'ont pas la possibilité ou le temps de rénover les maisons délabrées et ils préfèrent les louer aux groupes d'artistes et d'architectes qui travaillent effectivement pour restaurer la maison. La zone centrale est un périmètre protégé par la loi et, dans beaucoup de cas, il est difficile et coûteux d'y rénover une demeure. Il s'agit de se conformer aux avis de la mairie ou d'autres institutions publiques et d'appliquer des procédures spécifiques pour la conservation d'une maison.

21 C'est pourquoi, dans le cas que nous avons étudié, on ne trouve pas le phénomène de squatting - occupation de bâtiments -, présent dans d'autres capitales européennes comme Paris, par exemple. Lucian Sandu-Milea, architecte et artiste explique dans un entretien pour le journal en ligne, le petitparis.com, qu'il a été inspiré par le squatting à Paris, mais à Bucarest il a négocié un arrangement avec le propriétaire : " On cherchait une maison abandonnée de ce genre dans le quartier et un jour on est tombé sur le propriétaire. On a commencé à discuter avec lui et à lui présenter notre projet. La chance qu'on a eue c'est que lui, il cherchait à sauver la maison et nous une maison pour notre 
projet. [...] D’ailleurs, j'ai passé quelque temps à Paris pour y retrouver un ami, on était pas mal dans des endroits alternatifs. J'ai fait la connaissance de nombreux artistes qui travaillaient dans des squats à Tolbiac. Le côté débrouille et l'esprit de groupe m'a fortement inspiré pour créer Carol 53. » 9

Pour expliquer et illustrer la vie de ces espaces privés-créatifs, nous proposons d'examiner quatre maisons exemplaires : Carol 53, Casa Jurnalistului, Home Mătăsari et Colivia-La Cage -. Les quatre maisons choisies ont eu une activité permanente et consistante dans les dernières années et elles se sont impliquées dans l'organisation d'événements artistiques, civiques et sociaux importants.

23 Carol 53 a été créé en 2012 par un groupe de jeunes architectes et étudiants en architecture. Ils ont trouvé une vieille maison, occupée par des sans-abris, délabrée et pleine de déchets. La maison avait été confisquée par les autorités communistes et nationalisée dans les années 1950. Le propriétaire, un architecte roumain réputé, a pu la récupérer grâce à une décision de justice, après la Révolution de décembre 1989. Il a eu de nombreuses difficultés avec les sans-abris qui ont occupé la maison et l'ont ruinée en quelques mois. Dans ce contexte, l'intervention du groupe des jeunes architectes a été salutaire ${ }^{10}$.

Figure 1. Répartition des délégations québécoises dans le monde en 2017. 


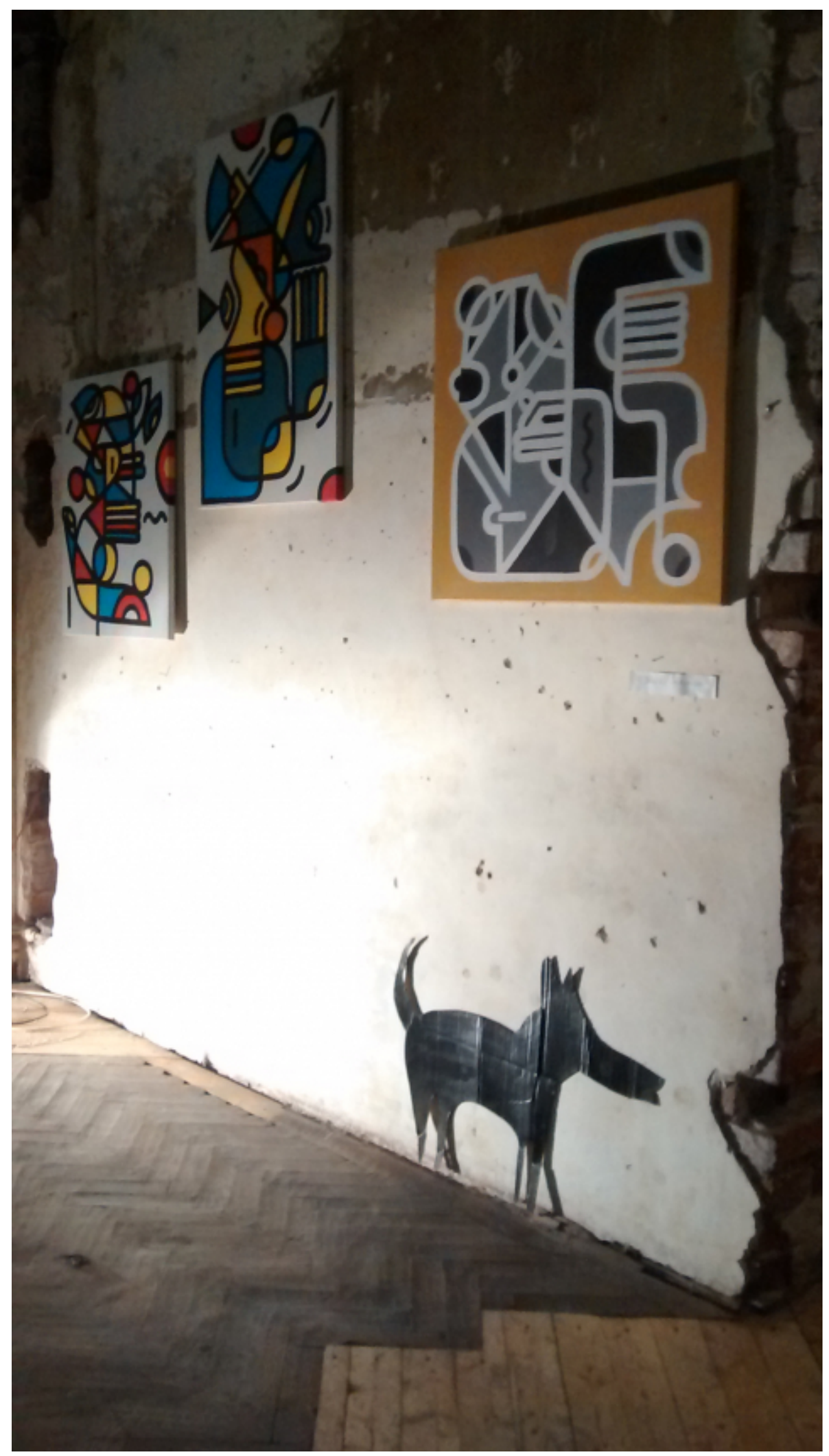

Source : Rapport annuel de gestion 2016-2017, ministère des Relations internationales et de la Francophonie.

Ceux-ci ont nettoyé la maison et l'ont restaurée en bonne partie : « La maison était dans un état déplorable, habitée par des sans domicile fixe qui avaient aussi beaucoup endommagé ses structures. [...] On a sorti quinze containers de poubelles ! On était un peu fous au début, on pensait qu'il y aurait moins de travail $»{ }^{11}$ L'espace a reçu une destination privée - quelques architectes et artistes habitaient et travaillaient ici et une destination 
publique - « nœud culturel ». Lucian Sandu-Milea, l'un des trois architectes qui s'occupent de cette maison, explique : " Nous produisons une culture émergente, authentique, sincère, peut-être naïve, qui appartient à Bucarest. Nous sommes un pôle culturel ici à Bucarest ${ }^{12}$. Plus de 300 événements culturels divers ont été organisés à Carol 53 dans les trois dernières années. De plus, un concours d'architecture s'est tenu en 2015, afin de sélectionner des jeunes architectes pour la restauration de la cour.

Casa Jurnalistului (la Maison du journaliste) a été créée à la fin de l'année 2011, par un jeune journaliste travaillant pour la presse écrite, Vlad Ursulean. Une véritable communauté de reporters et d'écrivains s'est développée autour de cet établissement. Plusieurs personnes vivent et travaillent dans la maison et d'autres participent régulièrement aux réunions de rédaction et aux fêtes qui y sont organisées. Les personnes interrogées pour cette recherche décrivent la maison comme étant une " maison ouverte », qui " ouvre l'esprit » (Surugiu, 2017). Elle s'est développée, selon leurs termes, comme une " communauté organique », remplie d'énergie, un "désordre contrôlé » ou une « folie ». Les journalistes acceptent volontairement la précarité du travail en échange de la liberté éditoriale. Les résidents reconnaissent l'influence du journalisme littéraire, considéré par la littérature académique comme l'un des futurs chemins du journalisme (Neveu, 2014). Le public est attiré par l'organisation bohème et par la production journalistique qui a une importante dimension littéraire. L'aspect bohème est accentué par l'organisation informelle du groupe, la maison ouverte aux visiteurs et les fêtes thématiques organisées régulièrement. Aussi, l'ambiguïté entre écriture journalistique et création littéraire qui est parfaitement associée à l'idéologie contemporaine de la créativité attire-t-elle également des jeunes qui admirent les esprits libres.

Ouverte en 2013, Colivia-La Cage - propose au public un espace qui est un studio photo, une salle de spectacle et une plateforme pour des projets civiques collaboratifs. La maison s'est définie comme une " expérimentation locative et lucrative ». Colivia abrite le bureau d'une association très connue, Funky Citizens, qui " lutte contre l'apathie sociale », en organisant des projets d'éducation civique. L'espace de Colivia a été découvert et utilisé par Daniel Vrăbioiu, un ingénieur ayant un penchant artistique - c'est un photographe passionné -. Il l'utilisait comme une sorte d'expérience artistique. Il a organisé la maison comme une maison ouverte, où il a habité et participé à l'organisation d'événements artistiques tels que "Homefest », petits concerts, débats sur des thèmes culturels et civiques etc. En mars 2015, il a cédé l'espace à l'association Funky Citizens, qui n'a pas préservé la dimension locative, mais seulement la dimension culturelle. Funky Citizens a continué de s'impliquer dans des projets culturels divers. Pour leur part, les artistes soutiennent cette association en prenant des messages civiques et en les traduisant dans leurs œuvres. Ainsi les travaux de Ramon Sadîc, considéré comme " l'artiste de la maison ", qui parvient à combiner les dimensions artistique et civique ${ }^{13}$. Colivia participe chaque année dans la Nuit des maisons, avec de petites expositions, installations artistiques, débats etc. L'événement est vu comme une possibilité d'élargir la « bulle », c'est-à-dire la communauté qui fréquente la maison ${ }^{14}$.

Figure 2. Les résidents de Casa jurnalistului sur la rue Viitorului à Bucarest - « Jurnalismul e în stradă (Le journalisme est dans la rue) » 


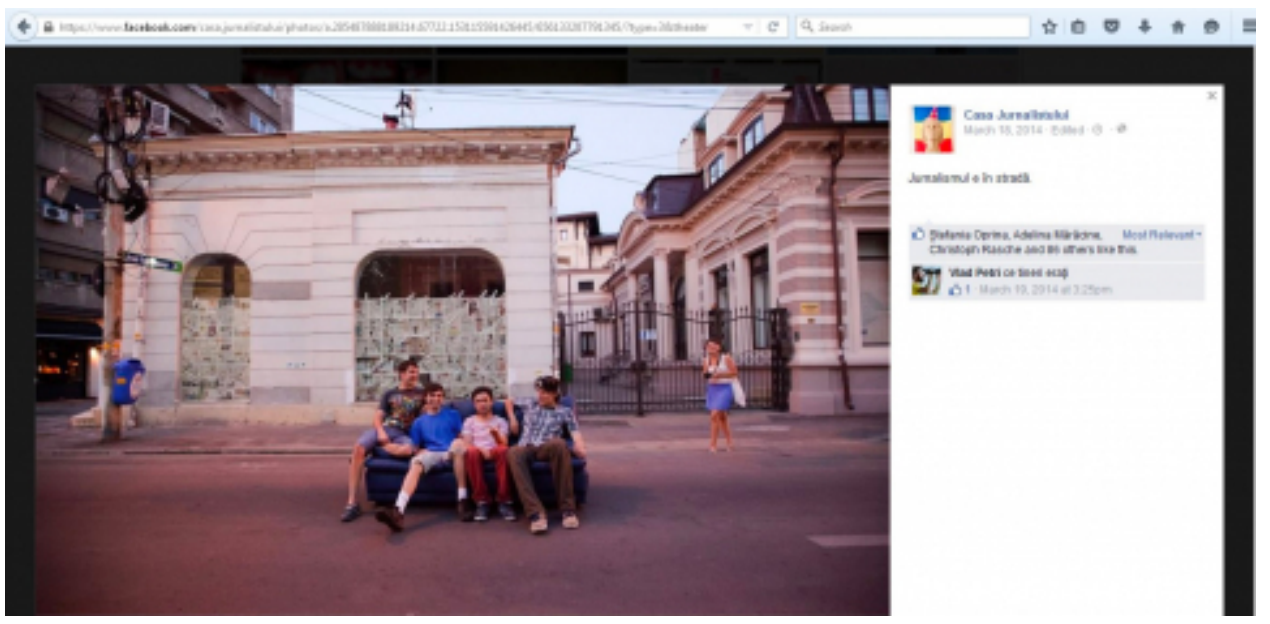

(C) FB Casa jurnalistului

Figure 3. Exposition de photos à Casa jurnalistului, Vlad \& Vlad la pas pe litoral (Vlad \& Vlad au bord de la Mer Noire), 2015

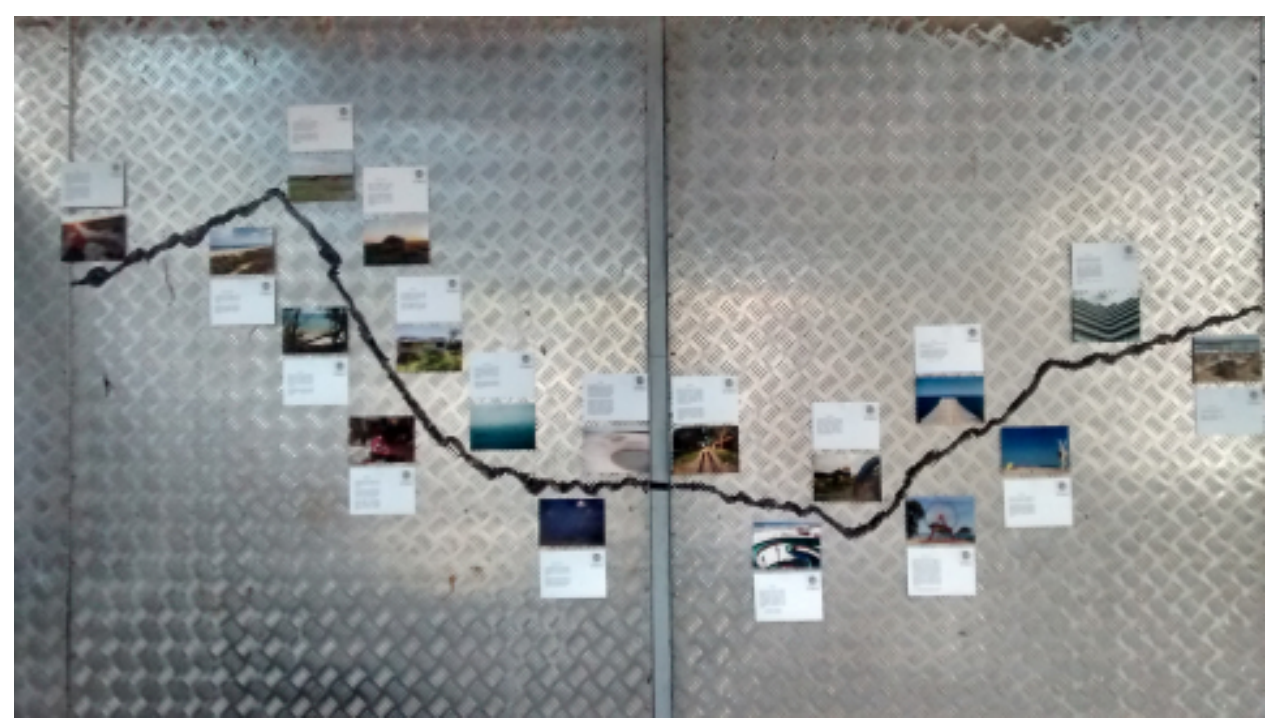

(c) Romina Surugiu

Figure 4. Intérieur de Casa Jurnalistului, tête d'une statue du poète national Mihai Eminescu avec chapeau russe et rouge à lèvres 


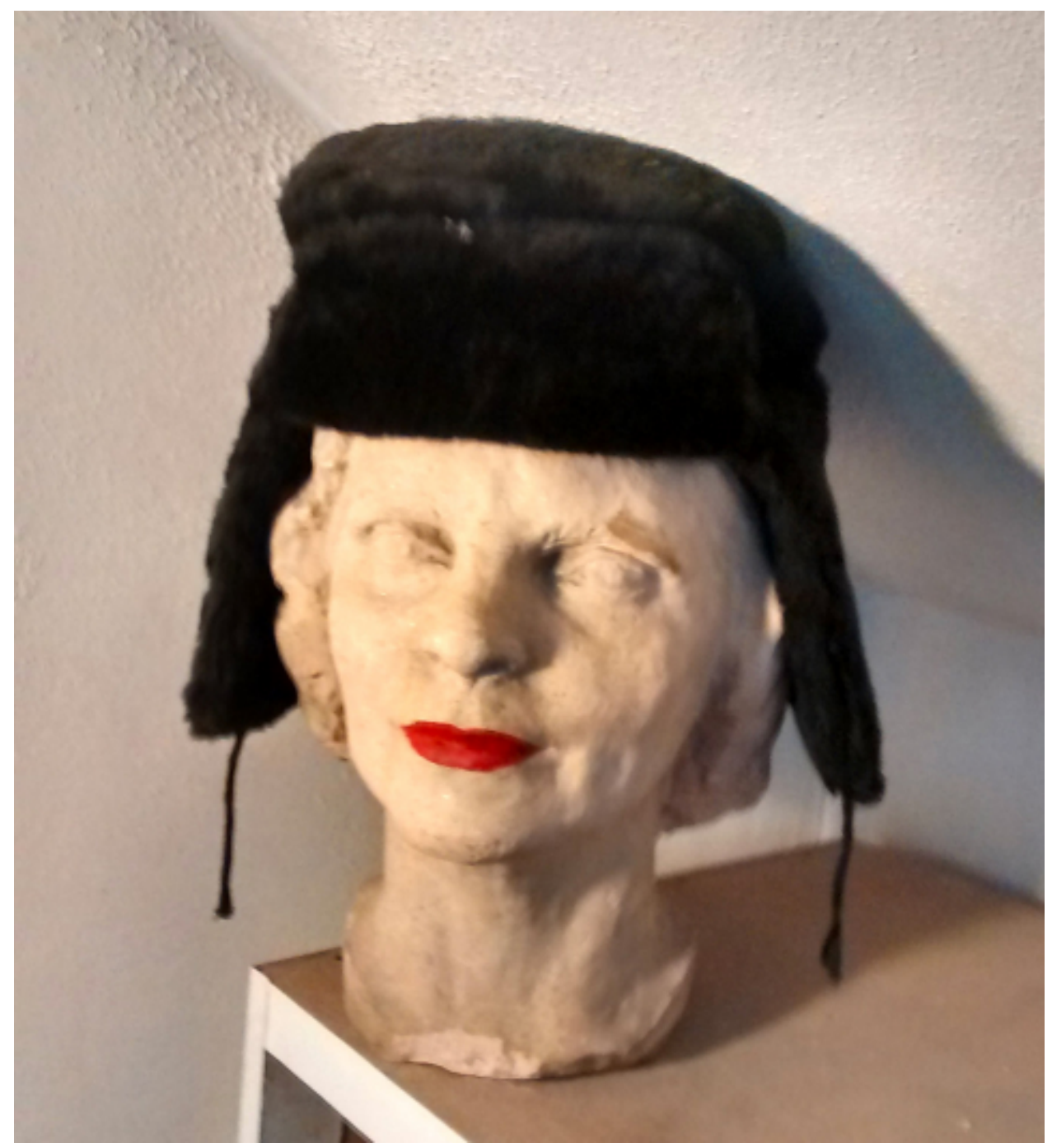

(C) Romina Surugiu

Figure 5. Intérieur de Colivia, avec un poêle en céramique original, vénitien, des années 1920 environ 


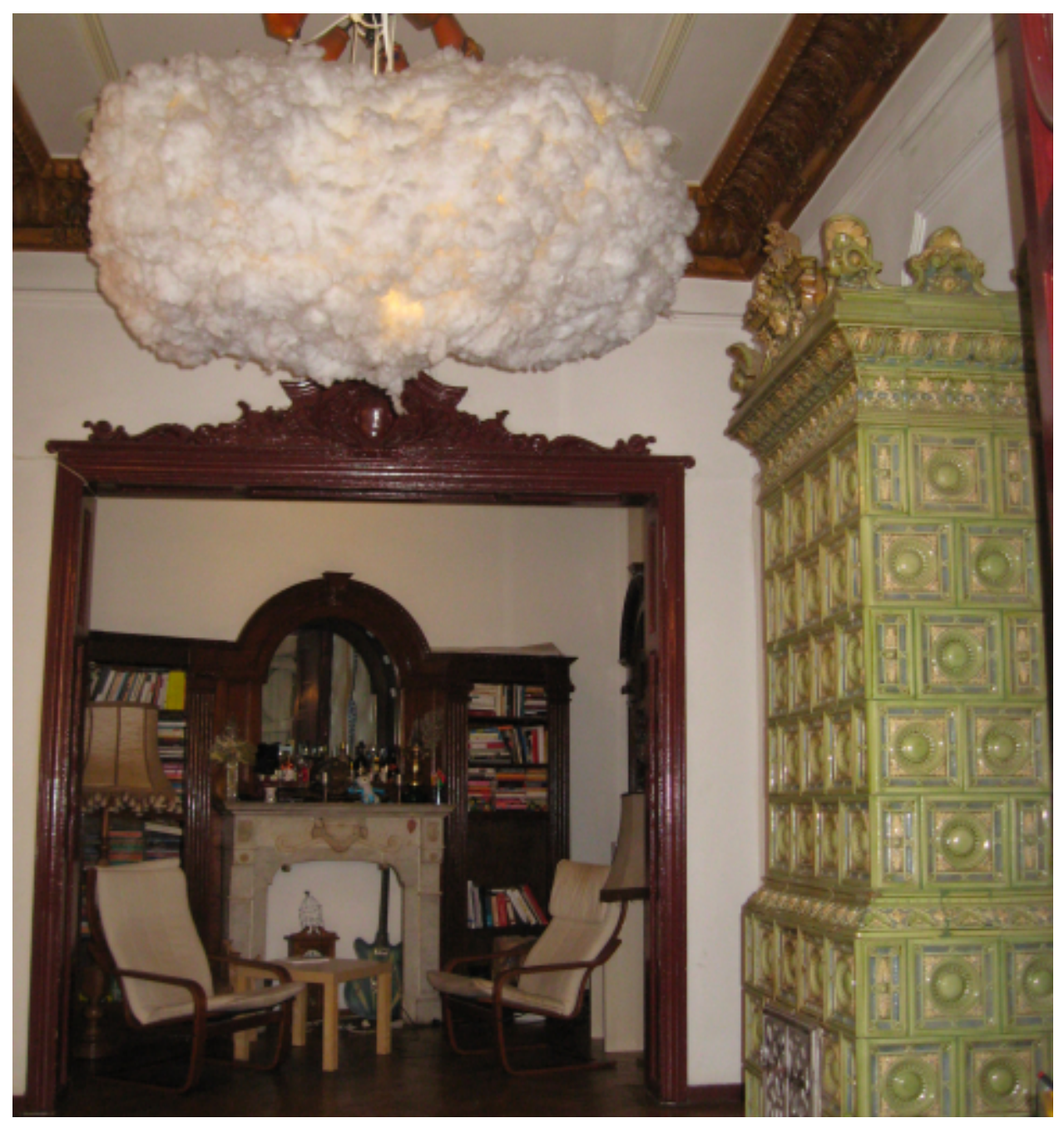

(C) Romina Surugiu

Créée en 2009, Home Mătăsari organise des expositions d'art urbain, de photographie, de graphisme et aussi des lancements de livres et des débats sur l'art. L'histoire de la maison est commune avec les autres demeures mentionnées ici : elle était délabrée. Le propriétaire l'a offerte pour un loyer convenable en échange des travaux de restauration effectués par les artistes. La maison en abrite huit, qui y résident et y travaillent, en se partageant les trois ateliers de création. Home Mătăsari a un programme informel pour ses artistes résidents. Chaque année, la maison offre à de jeunes artistes la possibilité d'y séjourner pendant quelques mois : la sélection est faite en fonction du projet artistique présenté. Il existe aussi des résidences permanentes pour les fondateurs.

Home Mtsri a également comme but de maintenir un " espace offline pour la socialisation créative et l'éducation informelle des étudiants ». L'activisme social des résidents et du public est encouragé par des actions culturelles, menées pour soutenir financièrement les familles des enfants malades de cancer. Iulian Văcărean, l'un des fondateurs, explique : "Home Mătăsari est apparue dans une communauté bucarestoise instable. La rue Mtsari est une rue où coexistaient la violence et la prostitution. Nous avons essayé de faire une insertion culturelle ici, par le logement dans une maison. Nous étions six artistes au commencement, venant de domaines tels que l'architecture, la photographie, la communication, le théâtre. Nous organisons des événements culturels atypiques : mini-théâtre, des récitals de poésie, des soirées-débat sur la lecture etc. Dans le cadre de Home Mătăsari nous avons mis au point un projet « Pași spre viață », qui aide financièrement les enfants malades de cancer hospitalisés à l'hôpital Marie Curie. Un événement très important pour notre groupe d'artistes est le festival « Femei pe Mătăsari » auquel participent de 20000 à 25000 personnes. Le festival, qui a lieu chaque année dans la dernière semaine de mai, veut changer les préjugés sur ce coin du centre- 
ville. Il y a donc beaucoup d'artistes qui se rassemblent dans une rue mal famée, afin de changer sa réputation ${ }^{15}$. »

Figure 6. Intérieur de Home Mătăsari, La Nuit des Maisons, 15 octobre 2016.

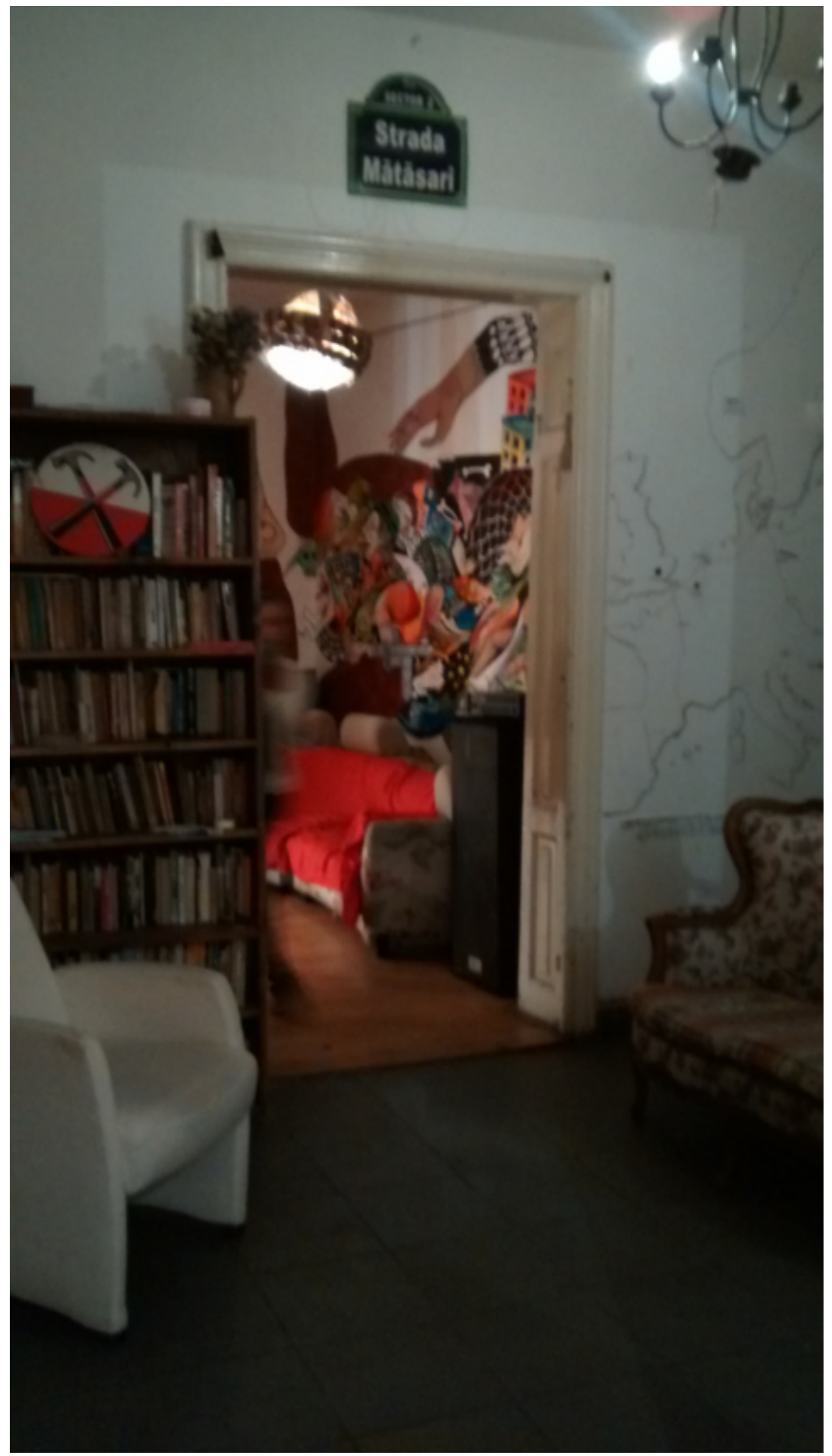

(c) Romina Surugiu 
L'initiative d'organiser des maisons pour les artistes n'existe pas seulement à Bucarest. À Iași, la plus importante cité de l'est de la Roumanie, on trouve Meru (Mișcarea Experimentală de Rezistență Urbană - Le Mouvement expérimental de résistance urbaine, qui a rénové en 2013 une demeure dans le centre-ville. Les artistes de Meru utilisent l'espace pour trois ateliers de création artistiques, pour une salle d'exposition et un café - qui est une sorte de moteur économique de cette entreprise culturelle indépendante -. Meru offre tous les six mois quatre résidences pour les artistes sélectionnés dans une compétition ouverte et transparente et projette d'agrandir le programme de résidence avec 8 à 10 artistes numériques de plus. Meru a organisé pendant les dernières quatre années plus de 120 événements culturels et collabore aussi avec d'autres maisons et espaces créatifs d'Iași : Galeria tranzit.ro, Borderline Art Space, The Grape, Hacker Space, Teatru Fix etc. ${ }^{16}$

30 À Banat, dans la zone Ciclova Montană - Ilidia - Socolari - Potoc, près de Timișoara, dans l'ouest de Roumanie, on trouve des artistes qui ont acheté des maisons dans les villages et les ont transformées en ateliers de création. Avec l'aide des autorités locales, ils ont organisé, en juillet 2017, le festival « Arta-n casa », L'Art dans la maison, qui a ouvert les portes des ateliers au public ${ }^{17}$.

\section{Un événement de médiation culturelle : la Nuit des maisons}

31 La Nuit des maisons est l'événement de médiation culturelle par excellence pour ces demeures. La première Nuit a eu lieu en 2014. Selon les dires des premiers organisateurs $^{18}$, elle a pris comme modèle les événements officiels de ce type, comme la Nuit blanche des galeries, qui a attiré le grand public dès la première édition (2007). Les galeries officielles, les ateliers de création et les hubs créatifs sont ouverts pour le public toute la nuit et organisent des événements culturels divers.

La Nuit des maisons se déroule sur le principe des portes ouvertes : les demeures peuvent être visitées pendant la nuit et le public a la possibilité de participer aux événements artistiques. On note aussi qu'un esprit ludique la caractérise. En effet, l'idée d'organiser cet événement est issue d'une blague - mimer et se moquer des choses « officielles » - et c'est pourquoi les organisateurs jouent avec le public, en combinant expositions sérieuses et événements divertissants, comme par exemple des fêtes où on écoute de la musique commerciale.

Figure 7. L'invitation pour la Nuit des maisons, 1re édition.

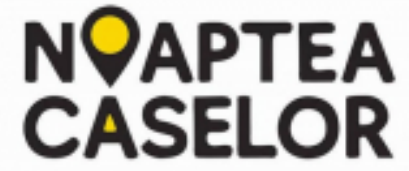

Un circuit al spațiilor domestic-culturale din Bucuresti.

27 SEPTEMBRIE 18:00-07:00

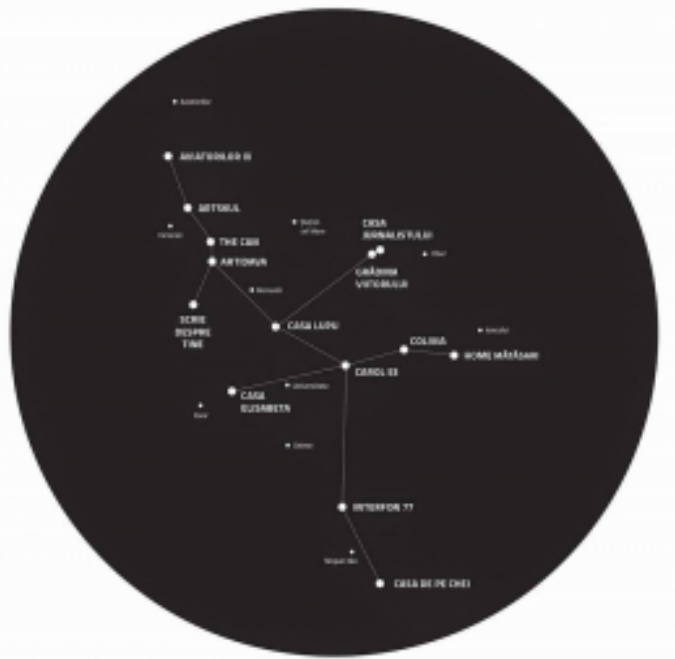


Organiser cette Nuit avait aussi pour objectif de voir combien d'espaces alternatifs existent et fonctionnent à Bucarest à un certain moment et combien subsistent d'une année sur l'autre. Ces espaces ont en effet un caractère éphémère suivant l'explication des organisateurs ${ }^{19}$.

Le 27 septembre 2014, à l'occasion de la première Nuit, 14 maisons ont participé : Galeria IX, Artskul, The Can, Artidava, Scrie despre tine, Casa Lupu, Gradina Viitorului, Casa jurnalistului, Home Matasari, Colivia, Carol 53, Casa Elisabeta, Interfon 77, Casa de pe Chei), le 26 septembre 2015. Pour la deuxième Nuit, il y avait 19 maisons : Home Matasari, Manasia Hub, Teatru 7, Cetatea Artelor, Vișini Hub, Plantelor, Grădina Sticlarilor, Casa Lupu, AvanPost, Insula 42, Learning House, Colivia, Casa Costa-Foru, Elisabeta, Casa jurnalistului, Centru Donici, Artskul, Carol 53, Incubator 107, et le 15 octobre 2016 à l'occasion de la troisième Nuit de nouveau 15 maisons : AICI, AvanPost, Carol 53, Casa jurnalistului, Casa Lupu, Cetatea Artelor, Colivia, CZVL12, EFDEN, Grădina Sticlarilor, Home Mătăsari, Insula 42, Manasia Hub, Modulab, The Room.

Plus d'un millier de personnes ont visité ces maisons, chaque année, selon l'estimation de Vlad Ursulean, de Casa Jurnalistului, l'un des organisateurs et auteur du programmemanifeste. Il a convaincu les représentants d'autres maisons des artistes d'ouvrir leurs demeures au public. L'événement a été promu sur Internet (http://noapteacaselor.ro/, @noaptea.caselor) et Casa Jurnalistului a préparé un dépliant avec des informations sur le circuit des maisons.

Chaque maison met en place divers événements pour cette occasion : des expositions de peinture, dessin ou photographie, des installations artistiques, des présentations de projets civiques, des spectacles musicaux et des fêtes. Les visiteurs reçoivent des gâteaux, du vin et des sandwiches. Les résidents présentent leurs maisons et les activités artistiques et civiques. Des ateliers pour les cyclistes ont également été mis en place. Pour les jeunes artistes, l'événement est une occasion d'exposer leur travail et d'interagir avec le public. Comme l'explique Andreea Toma, jeune peintre et exposante à Carol 53, en octobre 2016 : « j'avais besoin d'appréciation et d'approbation de la part du public pour savoir si mon art est sur le bon chemin. [...] Nous sommes tant d'artistes débutants, alors un événement comme celui-ci nous aide vraiment à nous faire connaître et devenir quelqu'un, surtout si tu ne trouves pas les moyens financiers de soutenir la création artistique ou si tu ne gagnes pas une bourse $»^{20}$.

Figure 8. Toile d'Andreea Toma, qui fait partie du projet medias, une parodie du « showbiz » roumain. Le projet était présenté au public à Carol 53, à l'occasion de la Nuit des maisons, 2016. 


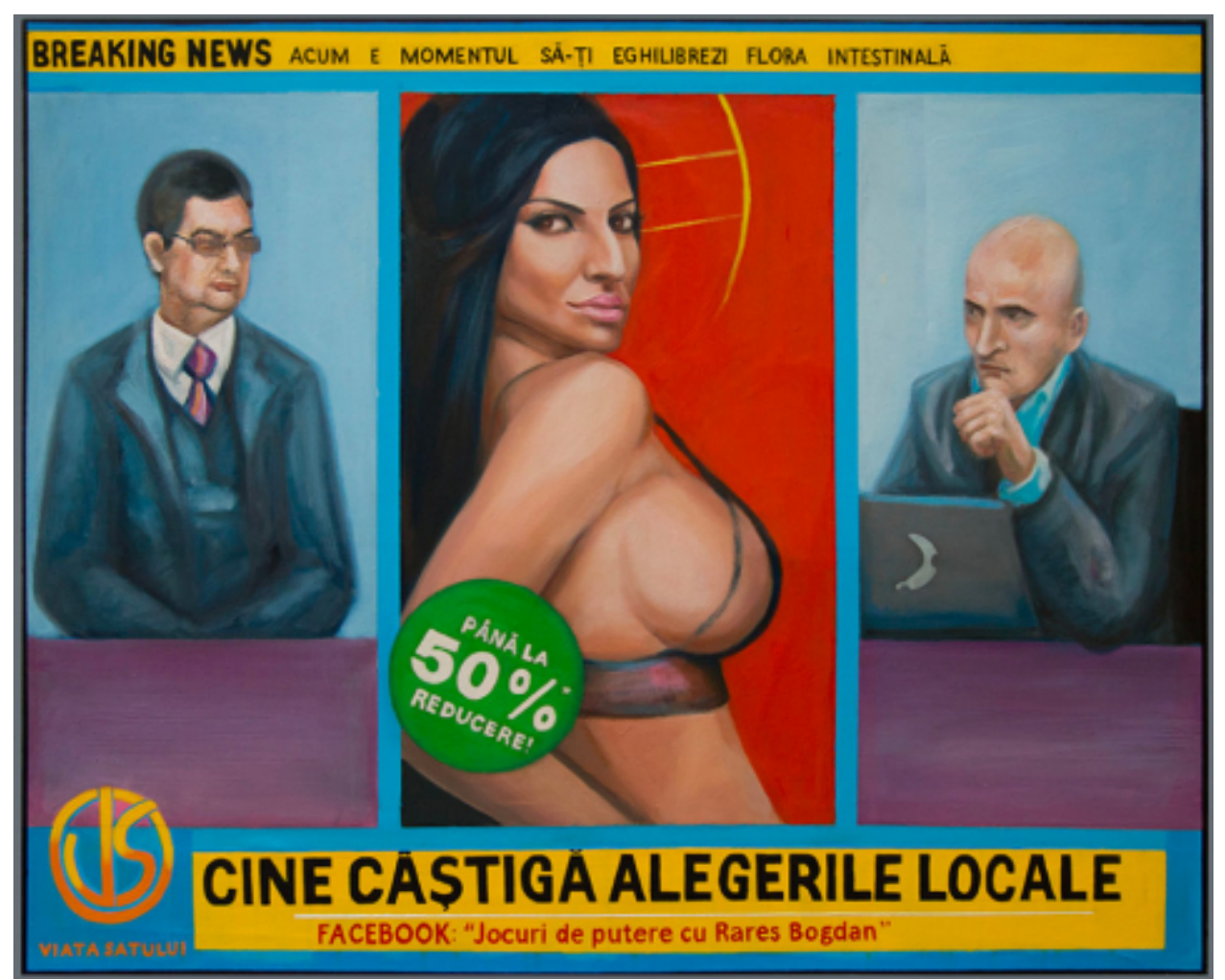

(c) Toma Andreea

\section{Conclusion}

Notre recherche a permis de présenter les maisons d'artistes indépendantes qui fonctionnent à Bucarest, sans appui institutionnel formel. Il s'agit d'espaces artistiques alternatifs ou off (au sens de Dumont et Vivant, 2016), où l'accent est mis sur l'expérimentation, sur le rejet sans équivoque de toute perspective commerciale ou industrielle, sur les liens d'amitié entre les artistes et sur l'importance vitale de l'emplacement/lieu. Considérant le caractère éphémère de ces entreprises culturelles, une question importante concerne l'avenir de ces espaces. L'avenir est aussi mentionné comme un élément inquiétant dans tous les entretiens faits pour cette recherche. Ainsi, l'activité dans les maisons est présentée comme work in progress, travaux en cours.

Ces maisons d'artistes sont l'avant-garde de la créativité et de l'innovation artistique dans la ville. Leur caractère bohème et la dimension locative représentent une rupture par rapport aux formules artistiques et institutionnelles obsolètes et une orientation vers l'authenticité, le vif, et la nouveauté, décrits comme " naïveté ». Pour en éviter le caractère éphémère, les artistes ne pratiquent pas le squatting. Ils cherchent des modalités pour interagir avec le public qui peut offrir un soutien important pour ces maisons, par le crowdfunding et par l'appréciation du point de vue esthétique.

À première vue, ces maisons sont des espaces embryonnaires pour le développement du bas vers le haut - de la dimension artistique de la ville, selon le modèle présenté par Richard Florida. Mais l'enquête de terrain a montré que les artistes ont choisi ces maisons pour la création artistique per se et pour exprimer une critique artiste et sociale (dans le sens de Boltanski et Chiapello, 1999). Les dimensions civique et artistique se mélangent dans un environnement bohème. Les artistes interviewés rejettent clairement l'appui institutionnel de la part de la mairie ou d'une autre autorité de l'État. Ils ont organisé ces lieux créatifs en delà des institutions formelles roumaines.

40 La médiation culturelle - sous la forme de fêtes, événements, portes ouvertes, spectacles - est plus qu'un instrument de reconnaissance de valeurs artistiques, mais une 
possibilité d'exprimer la critique artistique et sociale, de les rendre plus fortes dans la société, en vue d'équilibrer l'apathie sociale.

41 Ce travail pourrait avoir une portée explicative au niveau global, et non au seul niveau local. Elle montre comment les espaces culturels alternatifs s'efforcent de dépasser le caractère éphémère de leur condition et de s'intégrer dans la vie culturelle mainstream de la ville. En France, les lieux artistiques off se sont professionnalisés avec l'aide des autorités locales (Dumont et Vivant, 2016). En Roumanie, les maisons des artistes acquièrent une structure quasi-institutionnelle grâce au financement participatif et aux associations à but non lucratif. La question des subventions pourrait justifier de poursuivre ces travaux ${ }^{21}$.

\section{Bibliographie}

Becker (Howard S.). 1999. Propos sur l'art. Paris : L'Harmattan.

Bisenius-Penin (Carole) (sous la direction de). 2015. Résidence d'auteurs, création littéraire et médiations culturelles (1). À la recherche d'une cartographie. Nancy : PUN-Éditions universitaires de Lorraine.

Boltanski (Luc) \& Chiapello (Ève). 1999. Le Nouvel Esprit du capitalisme. Paris : Gallimard.

Cârneci (Magda). 2013. Artele plastice în România 1945-1989. Cu o addenda 1990-2010. Iași : Polirom.

Creswell (John W.). 2013. Qualitative Inquiry and Research Design. Choosing among Five Approaches. London : Sage.

Darchen (Sébastien) \& Tremblay (Diane-Gabrielle). 2010. " La thèse de la "classe créative". Revue des écrits et perspectives de recherche », p. 13-35 in La Classe créative selon Richard Florida. Un paradigme urbain plausible ? / sous la direction de Rémy Tremblay et Diane-Gabrielle Tremblay. Rennes : Presses universitaires de Rennes.

De Sardan (Jean-Pierre Olivier). 2014. La Rigueur du qualitatif. Les contraintes empiriques de l'interprétation socio-anthropologique. Louvain-la-Neuve : Academia Bruylant.

Dumont (Manon) \& Vivant (Elsa). 2016. « Du squat au marché publique. Trajectoire de professionnalisation des opérateurs de lieux artistiques off ». Réseaux, 6(200), p. 181-208.

Florida (Richard). 2002. The Rise of the Creative Class. And How it's Transforming Work, Leisure, Community, and Everyday Life. New York : Basic Books.

Florida (Richard). 2008. Who's Your City? How the Creative Economy is Making Where to Live the Most Important Decision of Your Life? New York : Basic Books.

Frunzaru (Valeriu) \& Ivan (Loredana). 2011. « Atitudinea faţă de muncă în subcultura creativă ». Calitatea Vieţii, 12 (1), p. 49-59.

Heinich (Nathalie). 2005. L'Élite artiste. Excellence et singularité en régime démocratique. Paris : Gallimard.

Hesmondhalgh (David) \& Baker (Sarah). 2011. Creative Labour. Media work in three cultural industries. London : Routledge.

Lakatoș (Andrei Eugen). 2017. Conversia în context. Despre regenerarea spațiilor industriale. București : Editura Fundației Arhitext Design.

Macrea-Toma (Ioana). 2009. Privileghenția. Instituții literare în comunismul românesc. ClujNapoca : Casa Cărții de Știință.

Neveu (Érik). 2014. " Revisiting Narrative Journalism as One of the Futures of Journalism ». Journalism Studies 15 (5), p. 533-542.

DOI : 10.1080/1461670X.2014.885683

Surugiu (Romina). 2017. «Freelancing as spectacular free labour : A case study on independent digital journalists in Romania », p. 183-195 in The Spectacle 2.o : Reading Debord in the Context of Digital Capitalism / sous la direction de Marco Briziarelli \& Emiliana Armano. London : Westminster University Press.

Tremblay (Rémy) \& Tremblay (Diane-Gabriele) (sous la direction de). 2010/2016. La Classe créative selon Richard Florida. Un paradigme urbain plausible? Rennes : Presses universitaires Rennes.

Villagordo (Éric). 2012. «Un sociologue en résidence artistique ». Culture \& Musées, 19, p. 147-168. DOI : 10.3406/pumus.2012.1652 
Vivant (Elsa). 2009. Qu'est-ce que la ville créative ? Paris : Presses universitaires de France.

DOI : 10.3917/puf.bouti.2009.01

\section{Notes}

1 Entretien avec Andreea Toma, le 18 août 2017.

2 Le texte complet est : «Certaines des choses les plus intéressantes à Bucarest se produisent récemment dans des maisons et des appartements où des communautés se sont réunies pour avoir des idées audacieuses.

Ceux qui ont commencé de grands projets sociaux et culturels ont frappé un mur de briques - autorités hostiles et environnement social apathique. Déçus, ils se sont réfugiés dans leurs espaces personnels. Mais leur esprit créatif a continué à fonctionner en privé.

Des amis ont commencé à s'impliquer, puis les amis de leurs amis, alors des communautés entières ont échangé des idées, faire des projets et se divertir. Leurs maisons se transforment peu à peu de logements en petits centres culturels.

Chacun d'entre eux est devenu un foyer d'innovation et d'esprit libre, en donnant un sens aux destinataires. Ensemble, ils peuvent créer un réseau culturel non structuré pour combler le vide laissé par des institutions formelles et laborieuses. Il est facile d'étouffer une institution mais un réseau informel est très résistant tant que ses communautés sont vivantes. La Nuit des maisons est un pont entre ces communautés. C'est un événement qui donne cohérence à une nouvelle tendance culturelle. Plus personnel, plus libre, plus efficace, plus cool.

Venez nous rejoindre!»

3 Ibid.

4 Entretien avec Andrei Eugen Lakatos, 15 août 2017.

5 Voir note 2.

6 Entretien avec Iulian Văcărean, 1 er août 2017.

7 Questionnaire par courriel, 20 août 2017.

8 Lucian Sandu-Milea explique la situation de Carol 53 : «Après la révolution de 1989, se développe un conflit entre deux tendances : il y a des personnes nostalgiques de la monarchie, qui accusent le communisme de leur avoir confisqué leurs propriétés, et des personnes nées pendant le régime communiste et qui ne connaissent que ça. La ville de Bucarest est très représentative de ce clivage. Nous, les jeunes, vivons ce temps où ces deux systèmes essayent de négocier, et où on essaye de dépasser ce conflit et de proposer autre chose, d'expérimenter tout en ne sachant pas où ça va mener. Ce projet en fait partie », Sarah Taher \& Grégory Rateau, « COUP DE COEUR - Carol 53, une maison abandonnée reprend vie ", www.lepetitjournal.com/Bucarest, Lundi 6 mars 2017, http://www.lepetitjournal.com/bucarest/communaute/coup-de-coeur-roumanie/272790-coup-decoeur-carol-53-une-maison-abandonnee-reprend-vie, consulté le 25 août 2017.

9 Ibid.

10 « Arhitecții din casa părăsită. Carol 53 », Film produit par Casa jurnalistului, disponible à https://www.youtube.com/watch?v=5IqLHw7zuRU, consulté le 26 août 2017.

11 Sarah Taher \& Grégory Rateau, « COUP DE COEUR - Carol 53, une maison abandonnée reprend vie ", www.lepetitjournal.com/Bucarest, Lundi 6 mars 2017, disponible à http://www.lepetitjournal.com/bucarest/communaute/coup-de-coeur-roumanie/272790-coup-decoeur-carol-53-une-maison-abandonnee-reprend-vie, consulté le 25 août 2017.

12 Entretien avec Lucian Sandu-Milea, 24 août 2017.

13 Entretien avec Elena Calistru, 22 août 2017.

14 Entretien avec Elena Calistru, 22 août 2017.

15 Entretien avec Iulian Văcărean, 1er août 2017.

16 Entretien par courriel avec Otilia Chitic, 28 Aout 2017.

17 Ioana Toma, «Arta'n casa : opt zile de scoala de vara cum nu s-a mai vazut », debanat.ro, le 30 juillet 2017, https://debanat.ro/2017/o7/artan-casa-opt-zile-de-scoala-de-vara-cum-nu-s-a-maivazut_198162.html, consulté le 26 août 2017.

18 Entretiens avec Lucian Sandu-Milea, le 24 août 2017 et avec Vlad Ursulean, le 27 octobre 2015.

19 Entretien avec Elena Calistru, 22 août 2017.

20 Entretien avec Andreea Toma, 18 août 2017.

21 Je tiens à remercier mes collègues Françoise Papa de l'université Grenoble Alpes, Costin Popescu de l'université de Bucarest et Andrei Eugen Lakatoș de l'université d'architecture et d'urbanisme 

projet.

\section{Table des illustrations}

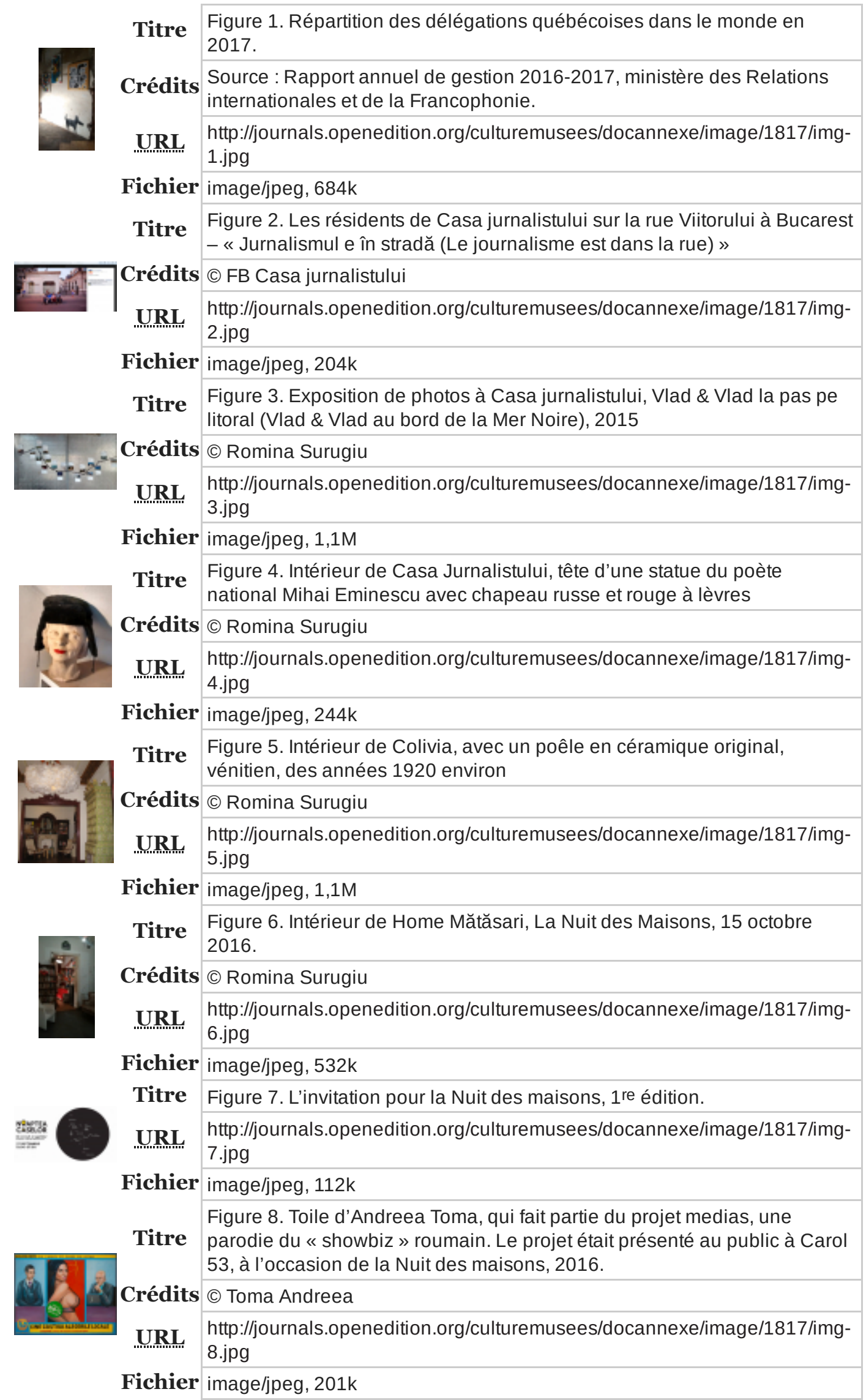




\section{Pour citer cet article}

Référence papier

Romina Surugiu, « Créer des espaces pour la création artistique et la médiation culturelle et civique : les maisons des jeunes artistes indépendants bucarestois », Culture \& Musées, 31 | 2018, 91-116.

Référence électronique Romina Surugiu, « Créer des espaces pour la création artistique et la médiation culturelle et civique : les maisons des jeunes artistes indépendants bucarestois », Culture \& Musées [En ligne], 31 | 2018, mis en ligne le 19 décembre 2018, consulté le 16 juin 2021. URL : http://journals.openedition.org/culturemusees/1817 ; DOI : https://doi.org/10.4000/culturemusees.1817

\section{Cet article est cité par}

- Rodríguez-Labajos, Beatriz. Ray, Isha. (2021) Six avenues for engendering creative environmentalism. Global Environmental Change, 68. DOI:

10.1016/j.gloenvcha.2021.102269

\section{Auteur}

\section{Romina Surugiu}

Romina Surugiu est maîtresse de conférences à la faculté de journalisme et de sciences de la communication de l'université de Bucarest. Ses domaines de recherche sont le journalisme, la production et la pratique professionnelle, le travail dans les industries culturelles, histoire des medias, les gender studies, la médiation culturelle et de la science. Parmi ses publications, on peut citer : «Freelancing as spectacular free labour : A case study on independent digital journalists in Romania », The Spectacle 2.0 : Reading Debord in the Context of Digital Capitalism I sous la direction de Marco Briziarelli \& Emiliana Armano. Westminster University Press, 2017 ; "The Fragile Professional Identities of Digital Journalists in Romania », Virtual Workers and the Global Labour Market, sous la direction de Juliet Webster \& Keith Randle, Palgrave Macmillan, 2016 ; « Pratiques journalistiques et nouvelles technologies. Recherche sur les jeunes journalistes roumaines » (co-authors Sorin Lazăr and Cristina Ilco), Cultures de la Communication, 1, 2016 ; « La médiation de la science. Analyse des expositions controversées des corps humains plastinés » (avec Viorica-Aura Paus), Patrimoine, création, culture. À l'intersection des dispositifs et des publics, sous la direction de Beatrice Fleury \& Jacques Walter, Paris, l'Harmattan, 2015. Elle dirige actuellement le projet de recherche : Télévisions et nations en « semi-périphérie » européenne : comment constituer une identité nationale par la télévision (1958-1980). Études de cas : la Roumanie, la Bulgarie et la Belgique, IFA-AUF 06/2016-2017. Courriel : romina.surugiu@fjsc.ro

\section{Droits d'auteur}

Culture \& Musées 